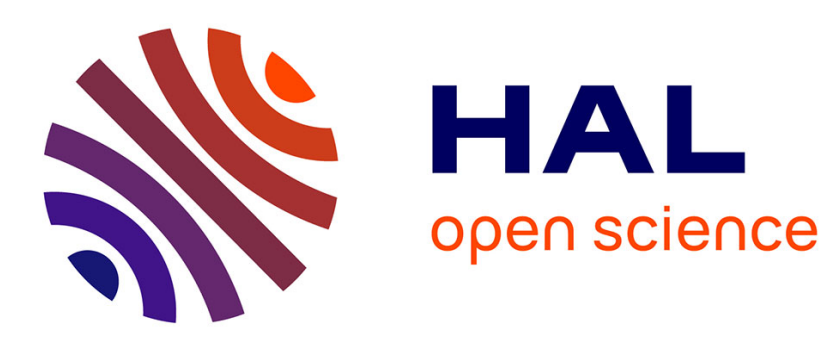

\title{
Confinement effect on the hydrated electron behaviour
} François-Xavier Coudert, Anne Boutin

\section{To cite this version:}

François-Xavier Coudert, Anne Boutin. Confinement effect on the hydrated electron behaviour.

Chemical Physics Letters, 2006, 428, pp.68-72. 10.1016/j.cplett.2006.07.023 . hal-00087416

\section{HAL Id: hal-00087416 https://hal.science/hal-00087416}

Submitted on 25 Jul 2006

HAL is a multi-disciplinary open access archive for the deposit and dissemination of scientific research documents, whether they are published or not. The documents may come from teaching and research institutions in France or abroad, or from public or private research centers.
L'archive ouverte pluridisciplinaire HAL, est destinée au dépôt et à la diffusion de documents scientifiques de niveau recherche, publiés ou non, émanant des établissements d'enseignement et de recherche français ou étrangers, des laboratoires publics ou privés. 


\title{
Confinement effect on the hydrated electron behaviour
}

\author{
François-Xavier Coudert and Anne Boutin*
}

July 3, 2006

\author{
Laboratoire de Chimie Physique, UMR 8000, \\ Université Paris-Sud 11, F-91405 Orsay Cedex, France \\ * Corresponding author: anne.boutin@lcp.u-psud.fr
}

\begin{abstract}
We report the first direct simulation of an excess hydrated electron confined in a zeolite nanopore by means of mixed quantum-classical molecular dynamics. The experimental dependence of the hydrated electron absorption spectrum maximum upon water loading in faujasites is reproduced. The diffusion of the confined hydrated electron is also studied and a prediction of the diffusion coefficient is provided.
\end{abstract}

\section{Introduction}

When a fluid is confined to spaces of molecular dimensions, a generic confinement effect is expected to take place in addition to the standard interface effect that arises from the interaction of the fluid molecules with the confined walls. Both effects may lead to drastic changes in the structure, dynamics and thermodynamics of the confined fluid, compared to its bulk counterpart. This is obviously expected to have a profound effect on any chemical event that may occur in the confined fluid. The question of how chemical reactions are perturbed by the size of the surrounding system is central in processes at the nanoscale. While an important amount of work was devoted in the past few years to the confinement effects on the thermodynamic behaviour of fluids, the study of chemical reactivity in confined media is still in its infancy due to the fact that many experimental techniques are difficult in a nanometer-confinement environment. [1,2]

In this context, radiolysis of confined liquids is a very interesting tool since ionizing radiation can initiate reaction in the whole system whatever its complexity. However both the confinement and interface effects influence the chemical reactions and these two effects are difficult to isolate from each other in the experiments. Even simple nanoporous materials have complex interfaces 
that strongly interact with the confined liquids (reactants and products) [3-5]. The interface effect can thus hide the hypothetical generic confinement effect or finite size effect. This is typically what happens when the reactivity of a solvated electron in water is studied in a zeolite [6-8]. Zeolites are crystalline aluminosilicate nanoporous materials that are widely used as selective catalysts and adsorbents [9]. The zeolitic materials used in industrial processes exhibit a low framework Si:Al ratio (typically between 1 and 3 ). The resulting high extra-framework cationic content makes these materials highly hydrophilic. This leads to high ion-exchange capacity that is used in particular for the removal of $\mathrm{Cs}^{+}, \mathrm{Sr}^{2+}$ and actinides from liquid nuclear waste [10] and the treatment of weapons-derived plutonium. [11] Furthermore, the importance of zeolites present in nuclear waste repositories extends far beyond cation exchange to phenomena affecting the entire thermohydrologic system. [12]

In the aluminosilicate zeolites studied by Thomas and coworkers [6] the hydrated electron strongly interacts with the extra-framework cations and the "interface" effect is likely to be dominant with respect to the confinement effect that we are looking for. Recently, solid-state chemists have concentrated in direct synthesis of all-silica zeolites $(\mathrm{Si}: \mathrm{Al}=\infty)$. [13,14] Recent reports, both experimental and theoretical, show that intrusion of liquid water inside these hydrophobic materials is indeed possible at pressures in the 50-200 MPa range. [15,16] These pure $\mathrm{SiO}_{2}$ crystalline materials can thus be considered as models of regular porous networks for studying the pure confinement effect since no aluminium atom in the structure nor extra-framework cations specifically interact with the adsorbed phase.

We report here mixed quantum-classical simulations (QCMD), successfully used to better understand the solvated electron-cations interactions in bulk water, $[17,18]$ applied to the study of the solvated electron in water confined in the pores of a siliceous faujasite. A schematic view of a faujasite supercage is represented in Figure 1. The porous network is made of large cavities of diameter $\sim 13 \AA$ that are tetrahedrally connected. In this widely open structure the water phase could be viewed as connected nanodroplets of 20-35 water molecules. The solvated electron was first observed in water by pulse radiolysis and transient optical absorption measurements in 1962, [19] and its reactivity has since been widely studied, mainly by pulse radiolysis. We report here results on optical, structural and dynamical properties of a solvated electron in confined water.

\section{Simulation technique}

We performed an adiabatic mixed quantum-classical molecular dynamics (QCMD) of an excess electron solvated in water confined in the nanopores of a zeolite. Only the excess electron was treated quantum mechanically, in the Born-Oppenheimer approximation. This approximation is well justified in the case of a fully relaxed hydrated electron where the energy gap between ground and excited states is fairly large $(\Delta E \simeq 2 \mathrm{eV})$. The forces acting on each classical degree 
of freedom are the Hellmann-Feynman forces as well as those arising from the empirical models used for the water and the zeolite framework. Water/water interactions are described by the SPC model [20], while the zeolite is described by a rigid framework modeled by single point charges and Lennard-Jones (LJ) centres with transferable parameters calibrated on adsorption isotherms and structural properties in various zeolites $[21,22]\left(q_{\mathrm{Si}}=+1.4 e, q_{\mathrm{O}}=-0.7 e, \sigma_{\mathrm{O}}=3.0 \AA\right.$ and $\epsilon_{\mathrm{O}}=95.53 \mathrm{~K}$; the Lennard-Jones cross parameters are calculated using the Lorentz-Berthelot mixing rule). A local pseudopotential developed by Turi and Borgis [23] is used to describe the excess electron-water interactions. This pseudopotential was optimized to reproduce the properties of an excess electron and a single water molecule and has been shown to successfully describe the interactions between the hydrated electron and water in the bulk at different thermodynamic conditions. $[23,24]$ The interaction between the electron and the zeolitic framework are considered to be purely coulombic. This last approximation is justified by the fact that the electron/zeolite interaction is mainly repulsive due to the negative partial charge of the oxygen atoms and thus only the long-distance, i.e. local part, of the pseudopotential is really significant in the simulations. Details of the implementation of the method, values of the water-excess electron parameters as well as a description of the cubic Gaussian basis set into which the electronic wavefunction is expanded can be found in Ref. [24].

A single cubic unit cell of a pure siliceous faujasite zeolite [25] (cell parameter $a \simeq 24.85 \AA$ ) is simulated using periodic boundary conditions and the Ewald summation technique is applied for the calculation of all long-range interactions. The simulations are performed in the NVT ensemble using the Nosé-Hoover thermostat to ensure isothermal conditions. The integration of the equations of motion for translation and rotation is performed using the Gear predictor-corrector algorithm, with a time step fixed at 0.5 fs. After $40 \mathrm{ps}$ of equilibration, production runs of $360 \mathrm{ps}$ are performed, from which are extracted all the properties presented in this work.

While the ground state electronic wavefunction is used to propagate the equations of motion, the first 20 excited states are also computed. The UV-Vis absorption spectrum of the solvated electron is then obtained as an histogram of the energy differences, $E_{n}-E_{0}$, weighted by the corresponding transition dipoles $\mu_{0 n}=\left\langle\Psi_{n}|\mu| \Psi_{0}\right\rangle$, or by using the cumulant expansion and the vibration-rotation decorrelation hypothesis. [26] Both methods lead to similar results. [27].

\section{Results and discussion}

We report the results of our simulations of a hydrated electron solvated in confined water, for 12 different water loadings ranging from 160 to 287 water molecules per unit cell. We have first performed Grand Canonical Monte Carlo simulations to calculate the water adsorption isotherm at $300 \mathrm{~K}$ following the methodology of Desbiens et al. [22]. The model faujasite is found to be hydrophobic and the intrusion pressure, estimated to be $60 \mathrm{MPa}$, is consistent with those calculated for other pure siliceous zeolites [28]. The maximum of adsorption of $\sim 287$ water molecules 
compares well with those measured experimentally of $\sim 260$ for sodium containing faujasites NaY and NaX. [29,30] At such loadings, all water molecules are located in the supercages of the zeolite only. This agrees with previous water adsorption studies in which it was found that water fills the sodalite cages only if there are cations in these small cages (i.e. in so called sites I'). [31] It is worth noticing that the water fills the porous volume homogeneously. For QCMD simulations, initial configurations with different numbers of water molecules were extracted from GCMC. In order to minimize the time needed for equilibration when we introduce the excess electron, we first pre-equilibrated a solvent cavity using a negative point charge.

The first section will present our results about the localization and diffusion of the solvated electron. The second section will focus on the UV-Vis absorption spectrum of the hydrated electron and its dependence on the water loading of the zeolite.

\section{Structure and diffusion}

The solvation structure of the confined hydrated electron is similar to that of the electron in bulk water, with one solvation shell, weakly defined in the oxygen and hydrogen Radial Distribution Functions. [24] This solvation shell features a number of molecule ranging from 4 at high water loading, which is the value of ambient-temperature bulk water, to 6 at low water loadings, with a less defined solvation sphere, as is the case for the electron in low-density bulk water. [32]

For all the 12 different water loadings simulated here, the hydrated electron is observed to move freely inside the zeolite supercages (diameter $\sim 13 \AA$ ) and the large twelve-membered rings connecting them (diameter $\sim 7.5 \AA$ ). During the 360 ps of each simulation, 10 trajectories show the hydrated electron jumping from one supercage to another, including 3 simulations during which the solvated electron visits three different supercages during the run. The crossing of the 12-ring windows is slow and the electron can spend up to $100 \mathrm{ps}$ in the window area between supercages, as can be seen on the example trajectory featured in Fig. 2 for 221 water molecules. This suggests that the free energy barrier associated with the crossing of the twelve-membered ring is of the order of $k_{B} T$, and the relatively small number of occurrences of this process is thus attributed to both the short timescale of our simulations and the slow intracage diffusion of the electron.

As a matter of fact, while the long-time intercage diffusion is not quantitatively accessible from our simulations beyond the above qualitative remarks, the short-time intracage diffusion of the hydrated electron is something that can be more precisely analyzed.

The values of the Mean Square Displacements $\left\langle\|\mathbf{r}(t)-\mathbf{r}(0)\|^{2}\right\rangle$ of the centre of the excess

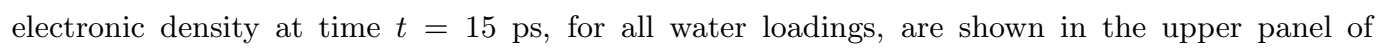
Figure 3, as well as the $\operatorname{MSD}(t=15 \mathrm{ps})$ for water molecules. These results indicate that the mobility of the hydrated electron decreases when the water loading increases.

Moreover, visual inspection of the MSD in the range $5 \mathrm{ps} \leq t \leq 15$ ps reveals a behaviour close

to a Brownian regime $\left(r^{2}(t) \propto t\right)$. The short-time self-diffusion coefficients $D_{\mathrm{s}}$ extracted from this 
region by Einstein's formula,

$$
\left\langle\|\mathbf{r}(t)-\mathbf{r}(0)\|^{2}\right\rangle \sim 6 D_{\mathrm{s}} t
$$

are shown in the lower panel of Figure 3, reflecting the same evolution upon water loading that the MSD at 15 ps. Also plotted in the lower panel of Fig. 3 are the self-diffusion coefficients of water for each water loading. The diffusion coefficients of both species are observed to have similar behaviour. Furthermore, the fluctuations observed for the hydrated electron diffusion coefficient at low loadings can be related to a noticeable deviation from the Brownian regime, as can be seen from the time evolution of the MSD (not shown here). The water diffusion coefficients show the same trend with loading that observed by Shirono et al. [33] in pure water adsorbed in sodium faujasite zeolites. The diffusion coefficient decreases with increasing number of adsorbed molecules at high loadings.

An important point is that the confined hydrated electron self-diffusion coefficients are 5 to 25 times smaller than that of the solvated electron in bulk water estimated with the same QCMD model, $D_{\mathrm{QCMD}}\left(e_{\mathrm{aq}}^{-}, \mathrm{bulk}\right) \simeq 6.810^{-5} \mathrm{~cm}^{2} \cdot \mathrm{s}^{-1}$. This value is slightly greater than the value estimated with the same electron/water pseudopotential using the polarisable pTIP4P water model,

$D_{\mathrm{QCMD}}^{\text {(pTIP4P) }}\left(e_{\mathrm{aq}}^{-}\right.$, bulk $) \simeq 4.010^{-5} \mathrm{~cm}^{2} . \mathrm{s}^{-1} .[26]$ The value of the diffusion coefficient in the faujasite at full water loading is computed in our study to be $0.2510^{-5} \mathrm{~cm}^{2} \cdot \mathrm{s}^{-1}$.

The experimental hydrated electron self-diffusion coefficient is only known in bulk water. In bulk phase at ambient conditions the calculated diffusion coefficient is 1.4 times larger for the hydrated electron than the experimental values $\left(D_{\exp }\left(e_{\mathrm{aq}}^{-}, \mathrm{bulk}\right) \simeq 4.910^{-5} \mathrm{~cm}^{2} \cdot \mathrm{s}^{-1}[34]\right)$. The SPC model also overestimates the diffusion coefficient by a factor of $1.7\left(D_{\mathrm{SPC}}\left(\mathrm{H}_{2} \mathrm{O}\right.\right.$, bulk $) \simeq$ $\left.4.310^{-5} \mathrm{~cm}^{2} \cdot \mathrm{s}^{-1}\right)$ and $D_{\exp }\left(\mathrm{H}_{2} \mathrm{O}\right.$, bulk $\left.) \simeq 2.510^{-5} \mathrm{~cm}^{2} \cdot \mathrm{s}^{-1}\right) .[35]$ We thus propose a corrected value for the self-diffusion coefficient of the electron in the hydrated faujasite of $0.20( \pm 0.05) 10^{-5} \mathrm{~cm}^{2} \cdot \mathrm{s}^{-1}$.

\section{Absorption spectrum}

The second part of our study of the solvated electron confined in the siliceous zeolite was to compute the UV-Vis absorption spectrum, which is experimentally one of its characteristic signatures. We observe that, while the spectra observed for different water loadings exhibit different shifts compared to that of a single solvated electron in bulk water, the overall shape of the absorption band and its half-maximum width are unchanged $\left(\Delta E_{1 / 2} \simeq 0.75 \mathrm{eV}\right)$. The evolution of the position of the maximum of the absorption spectrum with the water loading of the zeolite is shown in Figure 4, as well as experimental results for zeolites $\mathrm{Na}_{54} \mathrm{Y}$ and $\mathrm{Na}_{80} \mathrm{X}$. [6] Firstly, it can been seen that the shift observed from the hydrated electron in bulk water $\left(E_{\max } \simeq 1.95 \mathrm{eV}\right.$ in our simulations) can either be a red-shift or a blue-shift, depending on the number of water molecules. That fact as well as the general trend of the spectral shift is in good agreement with the experimental data, considering that the zeolites $\mathrm{Na}_{54} \mathrm{Y}$ and $\mathrm{Na}_{80} \mathrm{X}$ contain cations while we studied purely siliceous zeolites. 
While the absolute position of the absorption spectrum of the solvated electron at a given water loading depends on the presence of extra-framework cations in the zeolite [6], we suggest that the similarity in the evolution of the spectrum with respect to the number of water molecules present can be explained by a simple density effect. As was shown by previous studies, the position of the absorption spectrum of the solvated electron in bulk water is strongly influenced by the density of the liquid. [24,32] In particular, lower densities result in a red-shift of the spectrum, due to the larger size of the solvent cavity around the electron. To quantify this effect, we show in Figure 5 the correlation between the maximum of the absorption spectrum and the gyration radius of the hydrated electron for both bulk water at different densities and the zeolite at different water loadings. The two sets of data clearly coincides, strongly supporting the conclusion that the shift of the absorption spectrum of the hydrated electron in the zeolite upon different water loadings is essentially a density effect.

This density effect can be used to provide an indirect method of estimating a porous volume for the connected supercages. Using the relation established between the hydrated electron gyration radius and the local water density, we can estimate the density $\rho$ as a function of the number of water molecules present $n_{\mathrm{H}_{2} \mathrm{O}}$. The resulting curve can be fitted by a linear function, whose slope

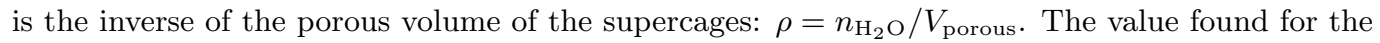

faujasite is $V_{\text {porous }} \simeq 7000 \AA^{3}$, in very good agreement with the supercages volume accessible to water estimated by Connoly methods [22], $V_{\text {porous }} \simeq 7150 \AA^{3}$. [28]

\section{Conclusions}

We report here, to our knowledge, the first direct simulation of an excess electron solvated by confined water inside nanopores. We presented the localization, diffusion and spectroscopic properties of the confined solvated electron, with a particular focus on the effect of water loading on the electron behaviour. The evolution of the absorption spectrum that we observe is in good agreement with available experimental data in different zeolites, and we propose the figure of $0.20( \pm 0.05) 10^{-5} \mathrm{~cm}^{2} . \mathrm{s}^{-1}$ for the intracage self-diffusion coefficient of the hydrated electron, which could be later used in mesoscopic simulations to improve our knowledge of the long-time dynamics in these systems.

Following this first encouraging results on a simple siliceous zeolite, work is under way to study systems for which experimental results are directly accessible, such as cationic zeolites $\mathrm{Na}_{54} \mathrm{Y}$ and $\mathrm{Na}_{80} \mathrm{X}$. The simulations reported here are but a first step in the use of mixed quantumclassical simulations to elucidate the complex behaviour of the solvated electron chemistry in confined fluids. 


\section{Acknowledgments}

Alain Fuchs is gratefully acknowledged for his support and numerous fruitful discussions. 


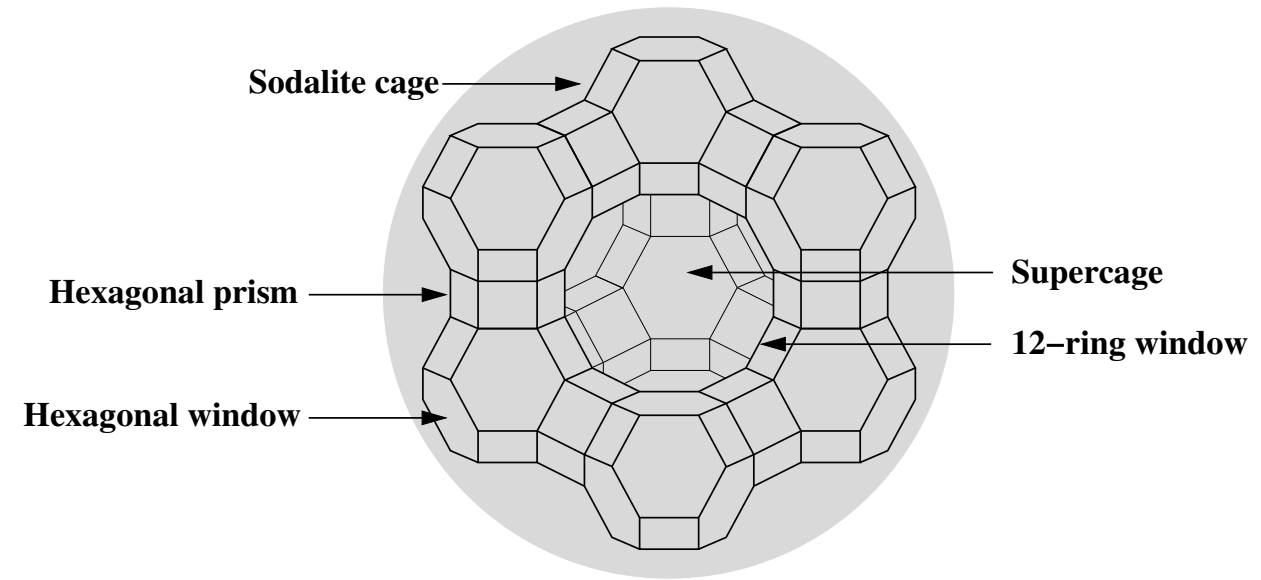

Figure 1: Schematic view of a faujasite supercage.

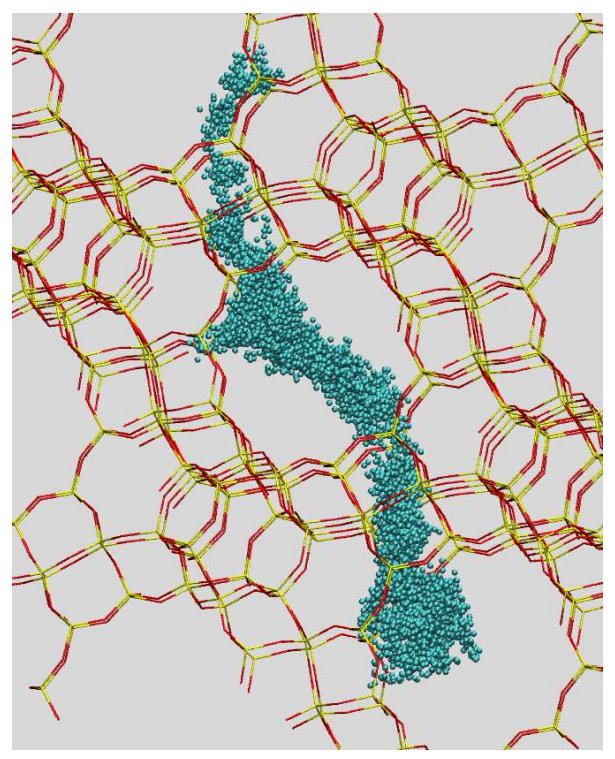

Figure 2: A 360 ps trajectory of the hydrated electron centre of mass (with 221 water molecules, not shown here). The electron visits three different supercages. 


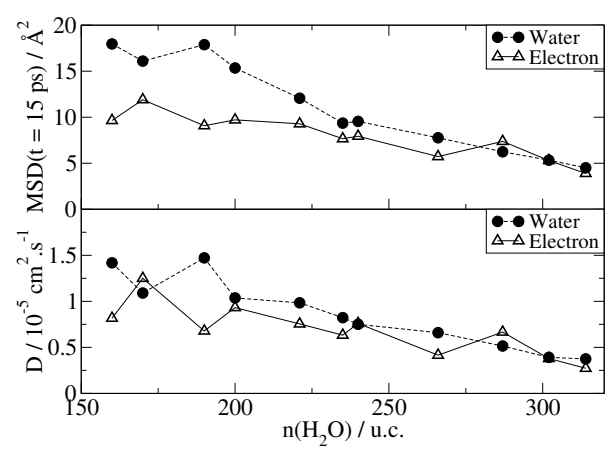

Figure 3: Upper panel: Mean Square Displacement (MSD) at $t=15$ ps for the water molecules (full circles) and the solvated electron (open triangles), as a function of the number of adsorbed water molecules, $n\left(\mathrm{H}_{2} \mathrm{O}\right)$. Lower panel: self-diffusion coefficient of water (circles) and the hydrated electron (triangles) as a function of $n\left(\mathrm{H}_{2} \mathrm{O}\right)$.

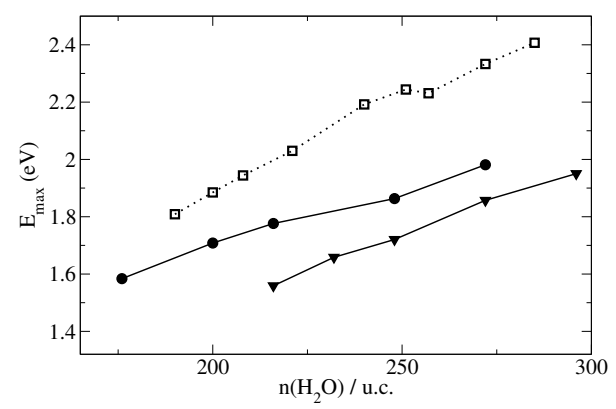

Figure 4: Position of the maximum of the UV-Vis absorption band of the hydrated electron, $E_{\max }$, as a function of the number of water molecules per unit cell, $n\left(\mathrm{H}_{2} \mathrm{O}\right)$ : simulation results for the siliceous faujasite (open squares), compared to experimental results for zeolites $\mathrm{Na}_{54} \mathrm{Y}$ (full circles) and $\mathrm{Na}_{80} \mathrm{X}$ (full triangles). [6].

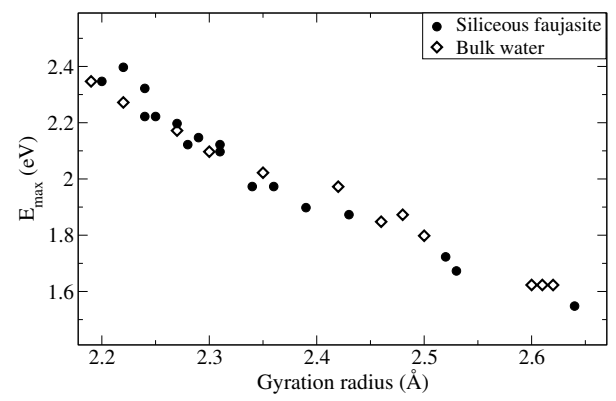

Figure 5: Correlation between the position of the maximum of the UV-Vis absorption band of the hydrated electron, $E_{\max }$, and its gyration radius for different water loadings in the zeolite (full circles) and different densities in bulk water (open diamonds). 


\section{References}

[1] Turro, N. J. Acc. Chem. Res. 2000, 33, 637-646.

[2] Hunt, P.; Worrall, D. R.; Wilkinson, F.; Batchelor, S. N. J. Am. Chem. Soc. 2002, 124, 8532-8533.

[3] Foley, S.; Rotureau, P.; Pin, S.; Baldacchino, G.; Renault, J.-P.; Mialocq, J.-C. Angew. Chem. Int. Ed. 2005, 44, 110-112.

[4] Caër, S. L.; Rotureau, P.; Brunet, F.; Charpentier, T.; Blain, G.; Renault, J. P.; Mialocq, J.-C. ChemPhysChem 2005, 6, 2585-2596.

[5] Rotureau, P.; Renault, J.-P.; Lebeau, B.; Patarin, J.; Mialocq, J.-C. Chem. Phys. Chem. 2005, 6, 1316-1323.

[6] Liu, X.; Zhang, G.; Thomas, J. K. J. Phys. Chem. B 1997, 101, 2182-2194.

[7] Zhang, G.; Liu, X.; Thomas, J. K. Radiat. Phys. Chem. 1998, 51, 135-152.

[8] Thomas, J. K. Chem. Rev. 2005, 105, 1683-1734.

[9] Auerbach, S. M.; Carrado, K. A.; Dutta, P. K.; Eds.. Handbook of Zeolite Science and Technology; Marcel Dekker, Inc. : New York, 2003.

[10] Pansini, M. Miner. Deposita 1996, 31, 563-575.

[11] Committee on Electrometallurgical Techniques for DOE Spent Fuel Treatment, Board on Chemical Sciences and Technology, National Research Council. An Evaluation of the Electrometallurgical Approach for Treatment of Excess Weapons Plutonium; 1996.

[12] Bish, D. L.; Vaniman, D. T.; Chipera, S. J.; Carey, J. W. Am. Mineral. 2003, 88, 1889-1902.

[13] Flanigen, E.; Bennett, J.; Grose, R.; Cohen, J.; Patton, R.; Kirchner, R.; Smith, J. Nature 1978, 271, 512-516.

[14] Corma, A.; Rey, F.; Rius, J.; Sabater, M. J.; Valencia, S. Nature 2004, 431, 287-290.

[15] Eroshenko, V.; Regis, R.-C.; Soulard, M.; Patarin, J. J. Am. Chem. Soc. 2001, 123, 81298130 .

[16] Desbiens, N.; Demachy, I.; Fuchs, A. H.; Kirsch-Rodeschini, H.; Soulard, M.; Patarin, J. Angew. Chem. Int. Ed. 2005, 44, 5310-5313.

[17] Spezia, R.; Nicolas, C.; Boutin, A.; Vuilleumier, R. Phys. Rev. Lett. 2003, 91, 208304.

[18] Coudert, F.-X.; Archirel, P.; Boutin, A. J. Phys. Chem. B 2006, 110, 607-615.

[19] Hart, E. J.; Boag, J. W. J. Am. Chem. Soc. 1962, 84, 4090.

[20] Berendsen, H. J. C.; Postma, J. P. M.; van Gunsteren, W. F.; Hermans, J. Interaction models for water in relation to protein hydratation; Reidel, Dordrecht, 1981. 
[21] Pascual, P.; Ungerer, P.; Tavitian, B.; Pernot, P.; Boutin, A. Phys. Chem. Chem. Phys 2003, 5,3684 .

[22] Desbiens, N.; Boutin, A.; Demachy, I. J. Phys. Chem. B 2005, 109, 24071-24076.

[23] Turi, L.; Borgis, D. J. Chem. Phys. 2002, 117, 6186.

[24] Nicolas, C.; Boutin, A.; Lévy, B.; Borgis, D. J. Chem. Phys. 2003, 118, 9689.

[25] Baerlocher, C.; Meier, W. M.; Olson, D. H. Atlas of zeolite framework types; 2001.

[26] Staib, A.; Borgis, D. J. Chem. Phys. 1995, 103, 2642.

[27] Spezia, R.; Nicolas, C.; Archirel, P.; Boutin, A. J. Chem. Phys. 2004, 120, 5261.

[28] Desbiens, N. private communication.

[29] Moïse, J.-C.; Bellat, J.-P.; Méthivier, A. Micropor. Mesopor. Mat. 2001, 43, 91-101.

[30] Dzhigit, O. M.; Kiselev, A. V.; Mikos, K. N.; Muttik, G. G. Russ. J. Phys. Chem. 1964, 38, 973-978.

[31] Beauvais, C.; Boutin, A.; Fuchs, A. H. ChemPhysChem 2004, 5, 1791-1793.

[32] Boutin, A.; Spezia, R.; Coudert, F.-X.; Mostafavi, M. Chem. Phys. Lett. 2005, 2005, 219-223.

[33] Shirono, K.; Endo, A.; Daiguji, H. J. Phys. Chem. B 2005, 109, 3446.

[34] Schmidt, K. H.; Han, P.; Bartels, D. M. J. Phys. Chem. 1992, 96, 199-206.

[35] Berendsen, H. J. C.; Grigera, J. R.; Straatsma, T. P. J. Phys. Chem. 1987, 91, 6269-6271. 